\title{
VLT/NACO near-infrared observations of the transient radio magnetar 1E 1547.0-5408 ${ }^{\star}$
}

\author{
R. P. Mignani ${ }^{1}$, N. Rea ${ }^{2}$, V. Testa ${ }^{3}$, G. L. Israel ${ }^{3}$, G. Marconi ${ }^{4}$, S. Mereghetti ${ }^{5}$, P. Jonker ${ }^{6}$, R. Turolla ${ }^{7}$, R. Perna $^{8}$, \\ S. Zane ${ }^{1}$, G. Lo Curto ${ }^{4}$, and S. Chaty ${ }^{9}$
}

\author{
1 Mullard Space Science Laboratory, University College London, Holmbury St. Mary, Dorking, Surrey RH5 6NT, UK \\ e-mail: rm2@mssl.ucl.ac.uk \\ 2 University of Amsterdam, Astronomical Institute "Anton Pannekoek", Kruislaan 403, 1098 SJ, Amsterdam, The Netherlands \\ 3 INAF - Osservatorio Astronomico di Roma, via Frascati 33, Monte Porzio Catone, Rome 00040, Italy \\ ${ }^{4}$ European Southern Observatory, Av. Alonso de Cordova 3107, Vitacura, Santiago, Chile \\ 5 INAF, Istituto di Astrofisica Spaziale, via Bassini 15, Milan 20133, Italy \\ ${ }^{6}$ SRON Netherlands Institute for Space Research, Sorbonnelaan, 2, 3584CA, Utrecht, The Netherlands \\ 7 Department of Physics, University of Padua, via Marzolo 8, Padua 35131, Italy \\ 8 JILA and Department of Astrophysical and Planetary Sciences, University of Colorado, 440 UCB, Boulder 80309, USA \\ 9 AIM-Astrophysique Interactions Multiéchelles (UMR 7158 CEA/CNRS/Université Paris 7 Denis Diderot), CEA Saclay, \\ DSM/IRFU/Service d'Astrophysique, Batiment 709, L'Orme des Merisiers, 91191 Gif-sur-Yvette Cedex, France
}

Received 31 October 2008 / Accepted 21 January 2009

\section{ABSTRACT}

\begin{abstract}
Context. Despite about a decade of observations, very little is known about the optical and infrared (IR) emission properties of the soft gamma-ray repeaters (SGRs) and of the anomalous X-ray pulsars (AXPs), the magnetar candidates, and about the physical processes which drive their emission at these wavelengths. This is mainly due to the limited number of identifications achieved so far, five in total, and to the sparse spectral coverage obtained from multi-band optical/IR photometry.

Aims. The aim of this work is to search for a likely candidate counterpart to the recently discovered transient radio AXP $1 \mathrm{E}$ 1547.0-5408.

Methods. We performed the first deep near-IR (NIR) observations ( $K_{\mathrm{s}}$ band) of 1E 1547.0-5408 with the Very Large Telescope (VLT) on three nights (July 8th, 12th, and August 17th), after the X-ray source rebrightening and during the subsequent decay reported around June 2007.

Results. We detected four objects within, or close to, the $3 \sigma$ radio position of 1E 1547.0-5408. The faintest of them (object 1 ) has a brightness $K \mathrm{~s}=20.27 \pm 0.05$, which would yield an unabsorbed X-ray-to-NIR flux ratio $F_{\mathrm{X}} / F_{K \mathrm{~s}} \sim 800$ for $1 \mathrm{E} 1547.0-5408$, i.e. on average lower than those derived for other magnetars. The non-detection of object 1 on the nights of July 8th and August 17th only allowed us to set an upper limit of $\Delta K_{\mathrm{s}} \sim 0.2$ on its NIR variability, which prevented us from searching for correlations with the radio or X-ray flux. We detected no other object at the radio position down to a limit of $K_{\mathrm{s}} \sim 21.7($ at $5 \sigma)$, computed in our deepest VLT image (July 12th).

Conclusions. From our observations we cannot confidently propose a NIR counterpart to 1E 1547.0-5408. More NIR observations of object 1, e.g. to determine its colors and to monitor variability, would be conclusive to determine whether or not it can be considered a plausible candidate.
\end{abstract}

Key words. stars: neutron - stars: pulsars: individual: 1E 1547.0-5408 - infrared: general

\section{Introduction}

Since the discovery of the first soft gamma-ray repeater (SGR 0526-66), almost exactly 30 years ago during the famous "March 5th 1979" event (Mazets et al. 1979), five SGRs have been discovered, the last one (SGR 0501+4516) in August 2008 (Barthelmy et al. 2008). After that of SGR 0526-66 came the discovery of the X-ray pulsar 1E 2259+586 (Gregory \& Fahlman 1980) which, after a decade, was recognized by Mereghetti \& Stella (1995) as a member of a new class of X-ray sources, the anomalous X-ray pulsars (AXPs). Nine AXPs since have been discovered plus one candidate (see Mereghetti 2008, for an updated review). Both SGRs and AXPs are believed to be

* Based on observations collected at the European Southern Observatory (ESO), Paranal, Chile under programme ID 079.D-0441(A). magnetars, isolated neutron stars with magnetic fields above the quantum limit $\left(4.14 \times 10^{13} \mathrm{G}\right)$, as suggested by the estimates of their surface dipolar magnetic field from spin-down measurements $\left(P=2-10 \mathrm{~s} ; \dot{P}=10^{-11}-10^{-10} \mathrm{~s} \mathrm{~s}^{-1}\right)$. At variance with other classes of X-ray pulsars, these objects are thought to be powered by energy stored in their huge magnetic fields (Duncan \& Thompson 1992; Thompson \& Duncan 1995), since their rotational energy is not a sufficient reservoir, and no evidence for accretion has been found so far.

While all magnetars are bright in the soft X-ray band, only a few of them are identified in the optical/near-infrared (NIR) domain. So far, the only SGR with an NIR counterpart is SGR 1806-20 (Israel et al. 2005; Kosugi et al. 2005). Among the AXPs, NIR counterparts have been identified for 4U 0142+61 (Hulleman et al. 2000, 2004), 1E 1048.1-5937 (Wang \& Chakrabarty 2002; Israel et al. 2002), XTE J1810-197 
(Israel et al. 2004; Rea et al. 2004), and 1E 2259+586 (Hulleman et al. 2001). The first two AXPs have also been detected in the optical, where their counterparts feature pulsations at the X-ray period (Kern \& Martin 2002; Dhillon et al. 2009). $4 \mathrm{U} 0142+61$ is also the only AXP/SGR detected in the mid-IR by Spitzer (Wang et al. 2006). The originally proposed NIR identification of 1RXS J170849-400910 (Israel et al. 2003) has now been ruled out by more recent observations (see, e.g. Testa et al. 2008, and references therein). Recently, NIR counterparts have been proposed for SGR 1900+14 and 1E 1841-045 (Testa et al. 2008) on the basis of possible long-term variability, although the identifications still need confirmation.

The optical/NIR emission spectra of magnetars are not fully characterized yet. This is mainly due to the high interstellar extinction which reduces the spectral coverage mostly to the NIR, while lower spatial resolution mid-IR observations are hampered by the local crowding. Moreover, in some cases the multi-band data-sets are derived from a compilation of measurements taken at different epochs which sample different source states and, thus, are not directly comparable. Interestingly, in all cases the counterpart fluxes fall a few orders of magnitude above the extrapolation in the optical/NIR domain of the blackbody component of the soft X-ray spectrum. It is not clear whether this NIR excess is due to a break in the magnetar spectrum or to the presence of an additional source of emission, possibly a debris disc (Perna et al. 2000), like that claimed around the AXP 4U 0142+61 (Wang et al. 2006). However, both for this source and for the AXP 1E 1048.1-5937 the detection of optical pulsations with pulsed fractions equal to the X-ray ones, or larger, and the lack of evidence for time lags between the X-ray and optical pulses (Kern \& Martin 2002; Dhillon et al. 2009) would argue against the optical/NIR emission being due to reprocessing of the X-ray radiation by a disc. A counter argument also comes by the observed erratic NIR variability of XTE J1810-197 (Camilo et al. 2007b; Testa et al. 2008), difficult to explain in the reprocessing disc scenario. However, a disc origin of the NIR emission is still considered a possibility (see, e.g. Ertan et al. 2007), and the upper limit on the polarisation of the NIR flux of $1 \mathrm{E}$ 1048.1-5937 (Israel et al., in preparation) does not provide compelling evidence for a non-thermal origin of its NIR emission. Interestingly, Mignani et al. (2007) pointed out that the high NIR emission efficiency of magnetars is likely associated with their huge magnetic fields.

One of the AXPs which still lack deep NIR observations is 1E 1547.0-5408. The source was detected by the Einstein X-ray observatory (Lamb \& Markert 1981), re-observed with the Advanced Satellite for Cosmology and Astrophysics (ASCA) by Sugizaki et al. (2001), and later on with XMM-Newton and Chandra. Its position at the centre of the candidate supernova remnant (SNR) G327.24-0.13, its high X-ray-to-NIR flux ratio, its X-ray flux decay by a factor of 10 over $\sim 25$ years, and its soft X-ray spectrum, prompted Gelfand \& Gaensler (2007) to propose 1E 1547.0-5408 as a candidate AXP. A radio pulsar (PSR J1550-5418) was then discovered by Camilo et al. (2007a), positionally coincident with $1 \mathrm{E}$ 1547.0-5408. The pulsar period $(P=2.069 \mathrm{~s})$ and period derivative $\left(\dot{P}=2.318 \times 10^{-11} \mathrm{~s} \mathrm{~s}^{-1}\right)$ yielded a spin-down age of 1.4 kyrs, a rotational energy loss $\dot{E}=1.0 \times 10^{35} \mathrm{erg} \mathrm{s}^{-1}$, and a magnetic field $B=2.2 \times 10^{14} \mathrm{G}$, thus confirming the magnetar interpretation. A distance of $9 \mathrm{kpc}$ was derived from the radio pulsar dispersion measure (DM), and highly $(\sim 100 \%)$ polarized radio emission at high frequency was also measured by Camilo et al. (2008). Monitoring Swift $X$-ray Telescope (XRT) observations of 1E 1547.0-5408 performed in 2007 June-July found that the source underwent a rebrightnening since its 2006 July-August minimum and was evolving to a new low state (Camilo et al. 2007a; Halpern et al. 2008). A long, follow-up XMM-Newton observation then led to the discovery of $\mathrm{X}$-ray pulsations at the radio period (Halpern et al. 2008). The lack of a radio detection before 2006 therefore suggests that the radio activity of the source is intrinsically related to its $\mathrm{X}$-ray emission state. This makes $1 \mathrm{E}$ 1547.0-5408 the second transient radio AXP discovered so far, after XTE J1808-197 (Camilo et al. 2006). NIR observations of 1E 1547.0-5408 were performed by Gelfand \& Gaensler (2007) with the $6.5 \mathrm{~m}$ Magellan telescope but they did not pinpoint any candidate counterpart down to $K_{\mathrm{s}} \sim 17.5$.

In this paper we present the results of the first deep NIR observations of 1E 1547.0-5408 performed with the Very Large Telescope (VLT). The observations are described in Sect. 2, together with the data analysis and calibration, while the results are presented and discussed in Sects. 3 and 4.

\section{Observations and data reduction}

\subsection{Observation description}

Thanks to our ESO Target of Opportunity program (ToO), we triggered VLT observations of 1E 1547.0-5408 right after the rebrightnening of the X-ray source, and subsequent decay, discovered by the Swift/XRT on 2007 June 22nd (Halpern et al. 2008). We observed 1E 1547.0-5408 in service mode on 2007 July 8th, 12th, and August 17th (Mignani et al. 2008) from the ESO Paranal Observatory with NAos COnica (NACO), the adaptive optics (AO) NIR imager and spectrometer mounted at the VLT Yepun telescope. In order to provide the best combination between angular resolution and sensitivity, we used the S27 camera which has a pixel size of 0.'027 and a field of view of $28^{\prime \prime} \times 28^{\prime \prime}$. As a reference for the AO correction we used the GSC-2 star S211131013236 $(V=14.3)$, located 30" away from our target. The visual (4500-10000 ̊) dichroic element and wavefront sensor were used. Unfortunately, only observations in the $K_{\mathrm{s}}$ band were executed as part of our program due to telescope scheduling constraints. To allow for subtraction of the variable NIR sky background, we split each integration in sequences of short dithered exposures with detector integration times (DIT) of $40 \mathrm{~s}$ and 4 exposures (NDIT) along each point of the random dithering pattern. The observation log is summarised in Table 1. In each night the target was observed close to zenith, with airmass always better than 1.2. Unfortunately, only for the July 12 th observation were seeing conditions good enough (i.e. below $0 .{ }^{\prime} 8$ ) to allow for an optimized use of the AO, while for the other nights the seeing never went below 1.'0. In particular, the observation of August 17th was affected by a seeing of $\sim 1$ 1.' $^{\prime}$. Because of its much lower image quality and shallower flux limit with respect to the others we did not use this observation in the subsequent analysis. Moreover, the observation of July 8th was affected by a strong wind above the pointing limit, and possibly by the presence of clouds. Sky conditions were photometric for the July 12th night only. Daytime (darks, lamp flat-fields) and night time calibration frames (twilight flat-fields) were taken daily as part of the NACO calibration plan. Standard stars from the Persson et al. (1998) fields were observed for photometric calibration at the beginning of each night.

\subsection{Data reduction and calibration}

The VLT data were processed through the ESO NACO data reduction pipeline ${ }^{1}$. Science frames were reduced with the

\footnotetext{
1 www.eso.org/observing/dfo/quality/NACO/pipeline
} 

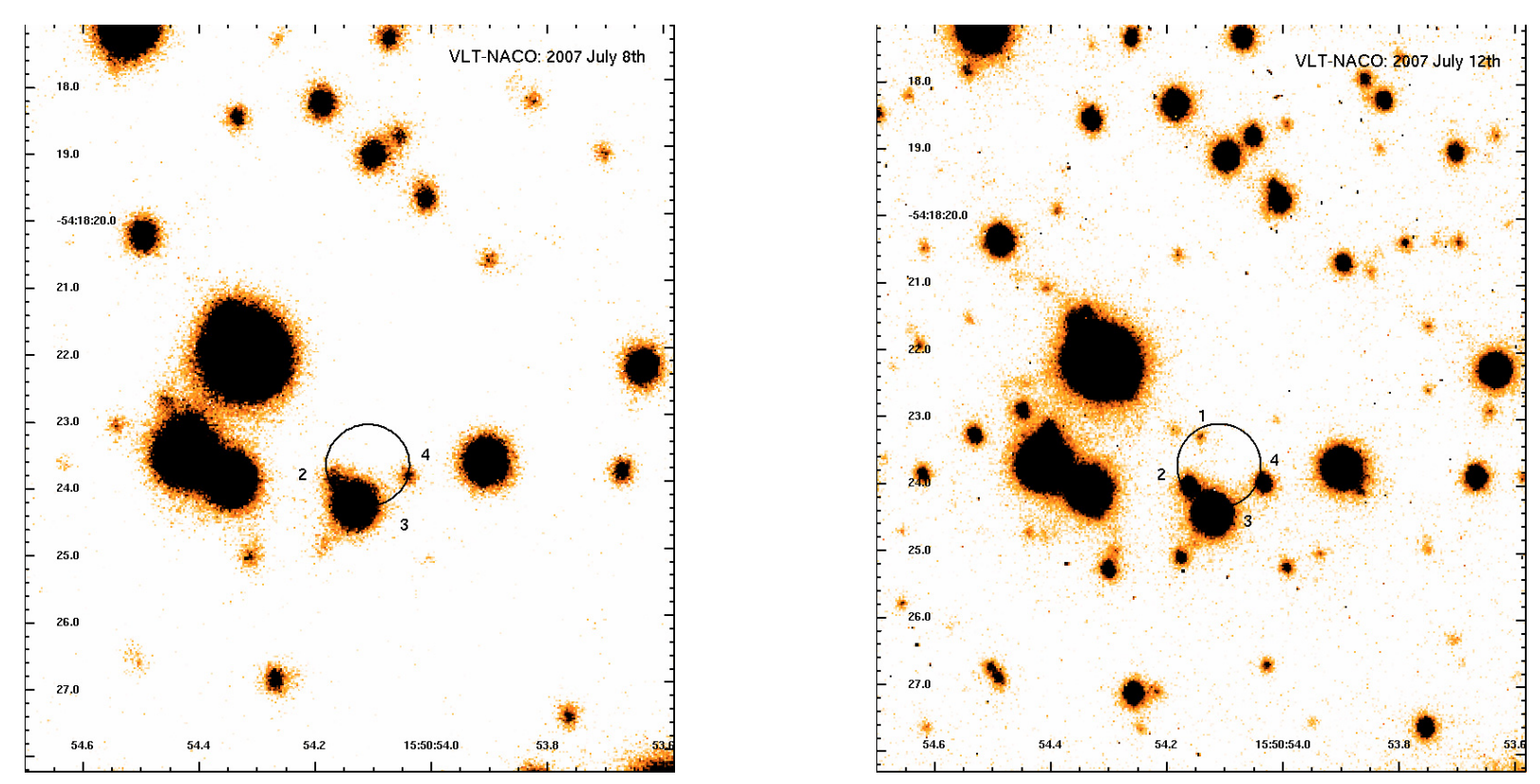

Fig. 1. VLT/NACO $K_{\mathrm{s}}$-band images of the 1E 1547.0-5408 field taken on 2007 July 8th $($ left) and 12th (right). North to the top, east to the left. The radius of the error circle $\left(0 \prime^{\prime} 63\right.$ at $\left.3 \sigma\right)$ accounts for the uncertainty on the radio coordinates of 1E 1547.0-5408 and for the overall error on our astrometric calibration (Sect. 2.2). Objects detected within, or close to, the radio error circle are labeled. The bright star south of it (\#3; $K \mathrm{~s}=16.23 \pm 0.03)$ is the object detected by Gelfand \& Gaensler (2007).

Table 1. Log of the VLT/NACO Ks-band observations of the $1 \mathrm{E}$ 1547.0-5408 field.

\begin{tabular}{ccccc}
\hline \hline $\begin{array}{c}\text { Epoch } \\
\text { (yyyy-mm-dd) }\end{array}$ & MJD & $\begin{array}{c}\text { Exp. time } \\
(\mathrm{s})\end{array}$ & $\begin{array}{c}\text { Seeing } \\
\left({ }^{\prime \prime}\right)\end{array}$ & Airmass \\
\hline $2007-07-08$ & 54289 & 1440 & 1.18 & 1.16 \\
$2007-07-12$ & 54293 & 1440 & 0.55 & 1.17 \\
$2007-08-17$ & 54329 & 1800 & 1.60 & 1.19 \\
\hline
\end{tabular}

Columns report the observing epoch and Modified Julian Date (MJD), the total exposure time $T=\mathrm{DIT} \times \operatorname{NDIT} \times N$, where $N$ is the number of nodes of the dithering pattern, and the average seeing and airmass during the observations.

produced master dark and flat-field frames and combined to correct for the exposure dithering and to produce cosmic-ray free and sky-subtracted images. We computed the astrometric calibration using the coordinates and positions of 6 stars selected from the 2MASS catalogue (Skrutskie et al. 2006). The pixel coordinates of the $2 M A S S$ stars (all non saturated and evenly distributed in the field) were measured by fitting their intensity profiles with a Gaussian function using the Graphical Astronomy and Image Analysis (GAIA) tool ${ }^{2}$. The fit to celestial coordinates was computed using the Starlink package ASTROM $^{3}$. The rms of the astrometric fit residuals was $\sigma_{\text {fit }} \approx 0$.'05 in the radial direction. We estimated the overall uncertainty of our astrometric solution by adding in quadrature the $\sigma_{\text {fit }}$ and the uncertainty $\sigma_{\mathrm{tr}}=\sqrt{3 / N_{s}} \sigma_{2 \text { MASS }}=0$.'14 with which we can register our field on the $2 M A S S$ reference frame. In this case, $\sigma_{2 \text { MASS }}=0.2$ is a conservative mean random radial error of the 2MASS coordinates (Skrutskie et al. 2006), $N_{\mathrm{s}}=6$ is the number of reference stars, and $\sqrt{3}$ accounts for the free parameters in the astrometric fit (e.g., Lattanzi et al. 1997). The uncertainty on the reference star centroids is below $0 .{ }^{\prime} 01$ and was neglected. This yields an overall uncertainty of our absolute astrometry of

\footnotetext{
2 star-www.dur.ac.uk/ pdraper/gaia/gaia.html

3 star-www.rl.ac.uk/Software/software.htm
}

0.15 . Since the $2 M A S S$ astrometry is tied to the international celestial reference frame (ICRF) with a $\sim 15$ mas accuracy, there is no significant shift between the radio and the NIR coordinates.

As a reference for the photometric calibration we assumed the airmass-corrected zero points provided by the NACO pipeline, computed through fixed aperture photometry. However, the zero point of July 8th shows a deviation of $\sim 0.5$ mag with respect to the mean of the measured values ${ }^{4}$, which has to be ascribed to the non-photometric conditions. We thus used the zero point measured on the night of July 12th $(23.24 \pm 0.02)$. We then performed a relative photometric calibration of the July 8th data by computing a linear fit between the measured instrumental magnitudes of $\sim 50$ sufficiently bright field stars detected in the field. For a direct comparison with the zero point computed by the NACO pipeline, we computed object fluxes through aperture photometry and we accounted for the aperture correction. We used the IRAF version of the Daophot package (Stetson 1992). The fit yielded an rms of $\sim 0.12$ mag which we assume as the systematic error of our relative photometry calibration.

\section{Results}

As a reference for the 1E 1547.0-5408 position we used the radio coordinates from Camilo et al. (2007a): $\alpha_{\mathrm{J} 2000}=$ $15^{\mathrm{h}} 50^{\mathrm{m}} 54.11^{\mathrm{s}}, \delta_{\mathrm{J} 2000}=-54^{\circ} 18^{\prime} 23^{\prime \prime} \cdot 7$, which have an absolute accuracy of $0.01^{\mathrm{s}}$ and $0 .{ }^{\prime} 1$ in right ascension and declination, respectively. The computed $3 \sigma$ radio error circle of $1 \mathrm{E}$ 1547.0-5408 is shown in Fig. 1, registered on the July 8th (left) and 12th (right) $N A C O K_{\mathrm{s}}$-band images. Four objects are detected within, or close to, the $3 \sigma$ radio error circle and are labeled in Fig. 1. The brightest one (\#3) is the object detected in the Magellan image of Gelfand \& Gaensler (2007). Three more objects are now detected in our image, one of which (\#2) is very close to the Gelfand \& Gaensler star and better resolved in the

\footnotetext{
4 www.eso.org/observing/dfo/quality/NACO/qc/zp_qc1. html
} 
Table 2. $K_{\mathrm{s}}$-band magnitudes of the candidate counterparts shown in Fig. 1, measured for the two nights.

\begin{tabular}{cccc}
\hline \hline Source ID & $\Delta r$ & 2007-07-08 & 2007-07-12 \\
\hline 1 & 0.50 & $\geq 20.1$ & $20.27 \pm 0.05$ \\
2 & 0.54 & $18.65 \pm 0.08$ & $18.51 \pm 0.03$ \\
3 & 0.76 & $16.22 \pm 0.05$ & $16.23 \pm 0.03$ \\
4 & 0.74 & $18.56 \pm 0.06$ & $18.54 \pm 0.03$ \\
\hline
\end{tabular}

The second column gives the source angular distance from the nominal radio position of $1 \mathrm{E} 1547.0-5408$. Only statistical errors $(1 \sigma)$ are listed. In all cases, the actual photometry error has to account for the zero point error $(0.02 \mathrm{mag})$ and, for the night of July 8 th, also for the rms of our relative photometry recalibration $(0.12 \mathrm{mag})$.

July 12th image thanks to its much better image quality. The faintest of the four objects (\#1), is detected in the July 12th image only. This is by far the deepest of the two, as shown by the difference in the number of objects detected in the field. The angular distance of objects \#1-4 from the nominal radio position is reported in the second column of Table 1 . We note that only objects $\# 1$ and 2 are detected within the $3 \sigma$ radio error circle. Although the not so tight positional coincidence argues against an association with the X-ray source, it does not formally rule it out.

We measured the magnitudes of objects \#1-4 through PSF photometry with Daophot, to better resolve the emission from object \#2 which is partially blended with object \#3 even in the best image-quality data of July 12 th. The results of our photometry for the two nights are shown in Table 2, for comparison. We note that the measured flux of object \#3 $\left(K_{\mathrm{s}}=16.23 \pm 0.03\right)$ is slightly lower than that reported by Gelfand \& Gaensler (2007) who gave $K_{\mathrm{s}}=15.9 \pm 0.2$. This is most likely due to the fact that objects \#2 and \#3 are not resolved in their lower resolution images (see their Fig. 3), which yields a flux overestimation for object \#3. For objects \#2, as well as for 3 and 4, the measured magnitudes are consistent within the statistical errors and the accuracy of our relative photometry recalibration (see Sect. 2.2 and caption to Table 2). Thus, none of them is variable on the time span of our observations. For object \#1 $\left(K_{\mathrm{s}}=20.27 \pm 0.05\right)$ no direct variablity check is possible since it was not detected in the July 8th image. Since the night was non-photometric, its limiting magnitude estimate is quite uncertain. In particular, the residuals of our relative photometry calibrations show a relatively large scatter of $\sim 0.2$ mag for $K_{\mathrm{s}} \approx 20$, which we assume as the random error affecting the July 8th photometry at this flux level. Thus, accounting for the $0.12 \mathrm{mag}$ rms of the recalibration fit, our estimate is affected by an overall uncertainty of $\sim 0.25$ mag, which translates into a $5 \sigma$ limiting magnitude of $K \mathrm{~s} \sim 20.1$ for the July 8th image. This implies an upper limit on the object \#1 variability of $\Delta K_{\mathrm{s}} \sim 0.2$, accounting for the error of the July 12 th measurement. Since none of the objects close to the $1 \mathrm{E}$ $1547.0-5408$ position is variable, with the possible exception of $\# 1$, in the following we use their magnitudes measured on the July 12 th image. We measured its $5 \sigma$ limiting magnitude to be $K \mathrm{~s} \sim 21.7$.

\section{Discussion}

The lack of color information does not enable us to determine which of the objects detected within the $3 \sigma$ radio error circle (Fig. 1) is a plausible candidate counterpart to 1E 1547.0-5408. While object \#2, as well as 3 and 4, is clearly non-variable between 2007 July 8th and 12th, object \#1 might still feature a tiny variability $\left(\Delta K_{\mathrm{s}} \leq 0.2\right)$. From the observed $\mathrm{X}$-ray flux decay rate

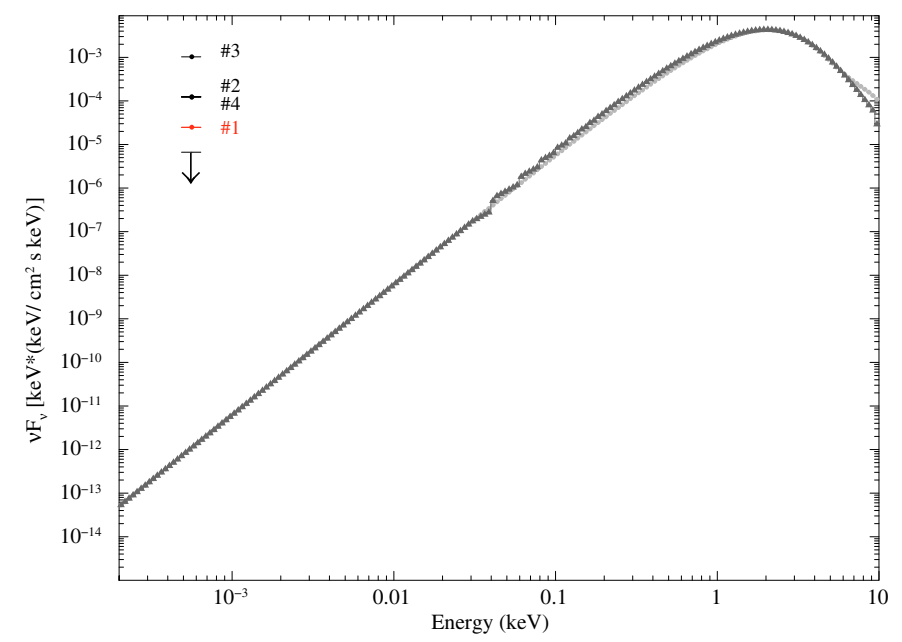

Fig. 2. Fits to the $S$ wift/XRT X-ray spectrum of 1E 1547.0-5408. To increase the signal-to-noise ratio, we merged the 2007 July 9th, 10th, and 16th datasets. Light and dark grey curves represent the fit with two blackbodies and a resonant cyclotron scattering (RCS) model, respectively (see text for details). We did not plot the blackbody plus powerlaw model since the latter component diverges when extrapolated to the optical/NIR domain. The dereddened $K_{\mathrm{s}}$-band flux of object \#1 (red) and 2-4 (black) and the $K_{\mathrm{s}}$-band flux upper limit derived from the July 12th observation are plotted.

of 1E 1547.0-5408 (Halpern et al. 2008), this would not rule out the variation expected if the NIR flux were to scale at the same rate as the X-ray one. Thus, we cannot use the comparison between NIR and X-ray variability to investigate a possible identification, as done for other magnetars.

We then considered the unabsorbed X-ray-to-NIR flux ratio as an alternative criterion. The X-ray spectrum of $1 \mathrm{E}$ 1547.0-5408 measured by Swift on 2007 July 9th, 10th, and 16th (Halpern et al. 2008), i.e. few days before and after our deeper NIR observation, can be fit by an absorbed blackbody plus power-law $\left(N_{\mathrm{H}}=3.2 \times 10^{22} \mathrm{~cm}^{-2}, k T=0.5 \mathrm{keV}\right.$ and $\Gamma=$ $2.93)$, two blackbodies $\left(N_{\mathrm{H}}=3.0 \times 10^{22} \mathrm{~cm}^{-2}, k T_{1}=0.5 \mathrm{keV}\right.$ and $\left.k T_{2}=1.3 \mathrm{keV}\right)$, or a resonant cyclotron scattering model $\left(N_{\mathrm{H}}=3.0 \times 10^{22} \mathrm{~cm}^{-2}, k T=0.5 \mathrm{keV}, \tau=1\right.$ and $\beta=0.24$; Rea et al. 2008a) ${ }^{5}$. As a reference, we plotted in Fig. 2 the best fit to the $S_{\text {wift }}$ XRT X-ray spectrum computed using two blackbodies and a resonant cyclotron scattering (RCS) model, together with the dereddened $K_{\mathrm{s}}$-band flux of objects \#1-4 and the $K_{\mathrm{s}}$-band flux upper limit. We computed the interstellar extinction correction in the $K_{\mathrm{S}}$ band using the hydrogen column density $N_{\mathrm{H}}$ derived from the spectral fits to the XRT data and applying the relation of Predhel \& Schmitt (1995) with the extinction coefficients of Fitzpatrick (1999).

Both the two blackbodies and the RCS spectral models yield an unabsorbed X-ray flux $F_{\mathrm{X}} \sim 5.8 \times 10^{-12} \mathrm{erg} \mathrm{cm}^{-2} \mathrm{~s}^{-1}$ in the $2-10 \mathrm{keV}$ energy range. The derived NIR flux $F_{K \mathrm{~s}}$ of object \#1 would imply an unabsorbed X-ray-to-NIR flux ratio $F_{\mathrm{X}} / F_{K \mathrm{~s}} \sim 800$ for $1 \mathrm{E} 1547.0-5408$, where $F_{K \text { s }}$ is integrated over the $K_{\mathrm{s}}$ band. We consistently recomputed the unabsorbed $F_{\mathrm{X}} / F_{K \mathrm{~s}}$ ratios for all magnetars with a secured NIR counterpart and we found a range of values which are on average a factor

\footnotetext{
5 The $N_{\mathrm{H}}$ values reported here are slightly different from those in Rea et al. (2008a) because, to be consistent with Halpern et al. (2008), we computed the $N_{\mathrm{H}}$ assuming the solar abundance values of Anders \& Grevesse (1989), while in Rea et al. (2008a) the more updated ones from Lodders (2003) have been used. Other spectral parameters are not affected.
} 
of 10 higher, or more. However, we note that there are several caveats in comparing $F_{\mathrm{X}} / F_{K \mathrm{~s}}$ ratios which, for a given source, are not always derived from contemporary X-ray and NIR observations and, for different sources, sample different X-ray states. Moreover, the computed X-ray fluxes can vary significantly, being much higher or lower depending on the assumed spectral model, while different NIR spectra can yield relatively larger $F_{K \mathrm{~s}}$ and, consequently, lower $F_{\mathrm{X}} / F_{K \mathrm{~s}}$. Thus, it is not obvious to firmly determine whether or not object \#1 can be considered a plausible candidate counterpart to $1 \mathrm{E}$ 1547.0-5408.

Given the crowding of the field, we also have to estimate the probability of a chance coincidence between the position of object \#1 and that of 1E 1547.0-5408. The chance coincidence probability can be estimated as $P=1-\exp \left(-\pi \rho r^{2}\right)$, where $r$ is the radius of the $3 \sigma$ radio error circle $\left(0^{\prime}{ }^{\prime} 63\right)$ and $\rho$ is the density of stellar objects in the NACO field of view. We computed $\rho$ from the object catalogue obtained by running Daophot, selecting star-like objects (ellipticity $e<0.2$ ) with magnitudes comparable to that of object \#1 and detected with at least $5 \sigma$ significance to filter out background fluctuations. In order to avoid systematic effects due to the degradation of the NACO PSF at large off-axis angles, we computed $P$ in the inner 13 '. $5 \times 13^{\prime \prime} .5$ region centred on the source position. We found $\rho \sim 0.03 \mathrm{arcsec}^{-2}$ which gives a chance coincidence probability $P \sim 0.04$, certainly not low enough to firmly claim, on the basis of the positional coincidence only, an association of object \#1 with $1 \mathrm{E}$ 1547.0-5408.

\section{Conclusions}

Using NACO at the VLT we performed the deepest NIR observations so far of the radio transient AXP 1E 1547.0-5408. We discovered a faint object (\#1; $K \mathrm{~s}=20.27 \pm 0.05)$ within $3 \sigma$ from the source radio position, which would imply an $F_{\mathrm{X}} / F_{K \mathrm{~s}}$ ratio of $\sim 800$, i.e. on average lower than those derived for other magnetars. Thus, we cannot confidently propose object \#1 as the NIR counterpart to 1E 1547.0-5408. More observations, to study its colors and to search for NIR variability correlated with the radio or X-ray one, would be conclusive to settle this issue. We note that at the time of writing of this manuscript a series of bursts from 1E 1547.0-5408 were detected by the Swift Burst Alert Telescope (BAT) on October 3, 2008 (Krimm et al. 2008; Rea et al. 2008b; Israel et al., in preparation), when the source was at the edge of the visibility window of ground-based telescopes and right before the Hubble Space Telescope (HST) was switched to safe mode. Follow-up Swift Ultraviolet Optical Telescope (UVOT) observations (Holland \& Krimm 2008) did not reveal any new candidate counterpart. On the other hand, if object \#1 is an unrelated field object, our observations would set a limit of $K_{\mathrm{s}} \sim 21.7$ on the brightness of the undetected $1 \mathrm{E}$ 1547.0-5408 counterpart, about 3 mag deeper than the previously reported limit.

Acknowledgements. R.P.M. acknowledges STFC for support through a Rolling Grant. N.R. is supported by an NWO Veni Fellowship, and thanks B. Gaensler, J. D. Gelfand, and F. Camilo for useful discussions on this source. S.Z. acknowledges STFC for support through an Advanced Fellowship.

\section{References}

Anders, E., \& Grevesse, N. 1989, Geochim. Cosmochim. Acta, 53, 197 Barthelmy, S. D., Baumgartner, W. H., Beardmore, A. P., et al. 2008, Astr. Tel., 1676

Camilo, F., Ransom, S. M., Halpern, J.P., et al. 2006, Nature, 442, 892

Camilo, F., Ransom, S. M., Halpern, J.P., et al. 2007a, ApJ, 666, L93

Camilo, F., Ransom, S. M., Penalver, J., et al. 2007b, ApJ, 669, 561

Camilo, F., Reynolds, J., \& Johnston, S. 2008, ApJ, 679, 681

Dhillon, V. S., Marsh, T. R., Littlefair, S. P., et al. 2009, MNRAS, in press [arXiv: 0901.1559v1]

Duncan, R. C., \& Thompson, C. 1992, ApJ, 392, L9

Ertan, Ü., Erkut, M. H., Eksi, K. Y., et al. 2007, ApJ, 657, 441

Fitzpatrick, E. L. 1999, PASP, 111, 63

Gelfand, J. D., \& Gaensler, B. M. 2007, ApJ, 667, 1111

Gregory, P. C., \& Fahlman, G. G. 1980, Nature, 287, 805

Halpern, J. P., Gotthelf, E. V., \& Reynolds, J. 2008, ApJ, 676, 1178

Holland, S., \& Krimm, H. A. 2008, GCN, 8325

Hulleman, F., van Kerkwijk, M. H., \& Kulkarni, S. R. 2000, Nature, 408, 68

Hulleman, F., Tennant, A. F., van Kerkwijk, M. H., et al. 2001, ApJ, 563, L49

Hulleman, F., van Kerkwijk, M. H., \& Kulkarni, S. R. 2004, A\&A, 416, 1037

Israel, G. L., Covino, S., Stella, L., et al. 2002, ApJ, 580, L143

Israel, G. L., Covino, S., Perna, R., et al. 2003, ApJ, 589, L93

Israel, G. L., Rea, N., Mangano, V., et al. 2004, ApJ, 603, L97

Israel, G. L., Covino, S., Mignani, R., et al. 2005, A\&A, 438, L1

Kern, B., \& Martin, C. 2002, Nature, 417, 527

Kosugi, G., Ogasawara, R., \& Terada, H. 2005, ApJ, 623, L125

Krimm, H. A., Beardmore, A. P., Gehrels, N., et al. 2008, GCN, 8312

Lamb, R. C., \& Markert, T. H. 1981, ApJ, 244, 94

Lattanzi, M. G., Capetti, A., \& Macchetto, F. D. 1997, A\&A, 318, 997

Lodders, K. 2003, ApJ, 591, 1220

Mazets, E. P., Golenetskii, S. V., Il'inskii, V. N., et al. 1979, Nature, 282, 587

Mereghetti, S. 2008, A\&ARv, 15, 225

Mereghetti, S., \& Stella, L. 1995, ApJ, 442, L17

Mignani, R. P., Perna, R., Rea, N., et al. 2007, A\&A, 471, 265

Mignani, R. P., Rea, N., Testa, V., et al. 2008, Astr. Tel., 1758

Perna, R., Hernquist, L., \& Narayan, R. 2000, ApJ, 541, 344

Predehl, P., \& Schmitt, J. H. M. M. 1995, A\&A, 293, 889

Rea, N., Zane, S., Turolla, R., Lyutikov, M., \& Götz, D. 2008a, ApJ, 686, 1245

Rea, N., Esposito, P., \& Krimm, H. A. 2008b, Astr. Tel, 1756

Skrutskie, M. F., Cutri, R. M., Stiening, R., et al. 2006, AJ, 131, 1163

Stetson, P. B. 1992, Astronomical Data Analysis Software and Systems I, ASP Conf. Ser., 25, 297

Sugizaki, M., Mitsuda, K., Kaneda, H., et al. 2001, ApJS, 134, 77

Testa, V., Rea, N., Mignani, R. P., et al. 2008, A\&A, 482, 607

Thompson, C., \& Duncan, R. C. 1995, MNRAS, 275, 255

Wang, Z., \& Chakrabarty, D. 2002, ApJ, 579, L33

Wang, Z., Chakrabarty, D., \& Kaplan, D. L. 2006, Nature, 440, 772 